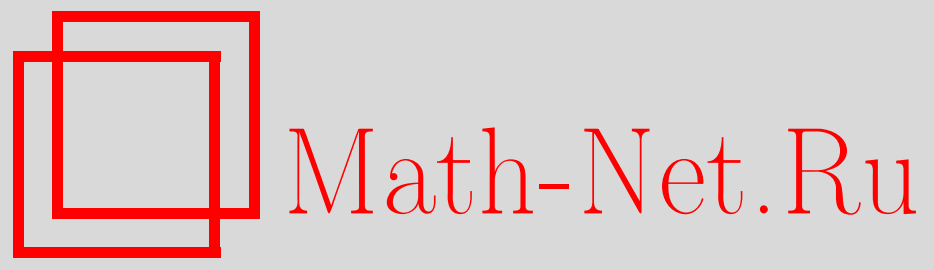

В. В. Высоцкий, Энергия и количество кластеров в стохастических системах неупругих притягивающихся частиц, Теория вероятн. и ее примен., 2005, том 50, выпуск 2, 241-265

DOI: https://doi.org/10.4213/tvp106

Использование Общероссийского математического портала Math-Net.Ru подразумевает, что вы прочитали и согласны с пользовательским соглашением

http://www . mathnet.ru/rus/agreement

Параметры загрузки:

IP : 34.229 .45 .116

26 апреля 2023 г., 12:51:27

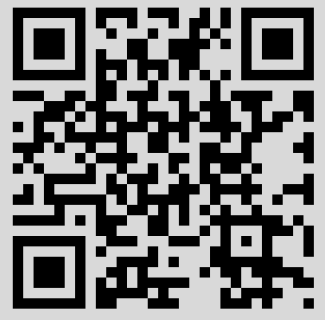




\title{
ЭНЕРГИЯ И КОЛИЧЕСТВО КЛАСТЕРОВ В СТОХАСТИЧЕСКИХ СИСТЕМАХ НЕУПРУГИХ ПРИТЯГИВАЮЩИХСЯ ЧАСТИЦ
}

\begin{abstract}
Мы рассматриваем одномерную модель гравитационного газа, частицы которого в начальный момент имеют случайные скорости и координаты. При столкновениях частицы слипаются, образуя «кластеры». В случае нулевых начальных скоростей («холодный газ») в терминах сходимости по вероятности изучено асимптотическое поведение количества кластеров $K_{n}(t)$ при $n \rightarrow \infty$, где $n$ означает число исходных частиц. Кроме того, исследуется асимптотика суммарной энергии газа $E_{n}(t)$. Здесь при ненулевых начальных скоростях («теплый газ») основным результатом является описание мгновенного «охлаждения» газа, т.е. $E_{n}(+0) \rightarrow 0$.
\end{abstract}

Ключевые слова и фразы: гравитационный газ, слипание частиц, неупругие соударения, система частиц, количество кластеров, энергия.

1. Введение. В этой статье мы изучаем одномерную модель гравитационного газа. Газ в начальный момент состоит из $n$ расположенных на прямой частиц, каждая из которых характеризуется массой, начальной координатой и начальной скоростью. Частицы начинают движение под действием сил взаимного тяготения. При столкновениях частицы слипаются, образуя новую частицу («жластер»), характеристики которой определяются законами сохранения массы и импульса. Движение частиц между столкновениями подчинено законам классической механики.

Слипание отдельных частиц в один большой кластер напоминает процесс образования звезды из космической пыли. Более подробно об использовании рассматриваемой модели в астрофизике можно узнать из статьи [1].

Нашей целью является изучение свойств газа при условии, что начальные положения и начальные скорости частиц являются случайными величинами, а число исходных частиц стремится к бесконечно-

* Санкт-Петербургский государственный университет; математико-механический факультет, Старый Петергоф, Библиотечная пл., 2, 198504 С.-Петербург, Россия; e-mail: vysotsky@vv9034.spb.edu 
сти. Различные предельные свойства таких систем рассмотрены в рабо$\operatorname{tax}[2]-[6]$. Мы исследуем поведение ранее не изученной величины $K_{n}(t)$, которая обозначает количество кластеров в системе. Также нас интересует $E_{n}(t)$ - энергия системы, т.е. сумма кинетических энергий всех кластеров.

Оказывается, что результаты абсолютно различны при рассмотрении моделей с нулевыми («холодный газ») и ненулевыми («тепльий газ») начальными скоростями. Мы докажем, что в холодном газе при любом $t \geqslant 0$

$$
\frac{K_{n}(t)}{n} \stackrel{\mathbf{P}}{\longrightarrow} f(t), \quad n \rightarrow \infty,
$$

где $f(t)$ - детерминированная функция; $f(t)=0$ при $t \geqslant 1$. Отсюда в любой фиксированный момент времени $t \in[0,1)$ среднее число частиц в одном кластере для холодного газа есть константа.

В случае теплого газа, наоборот, мгновенно образуются сколь угодно большие кластеры. За счет этого происходит «охлаждение», т.е. энергия газа мгновенно падает до нуля. В статье дано теоретическое обоснование этого феномена, который был эмпирически обнаружен в [5]. Кроме того, доказано, что и в холодном, и в теплом газе при любом $t \in(0,1)$

$$
E_{n}(t) \stackrel{\mathbf{P}}{\longrightarrow} \frac{t^{2}}{6}, \quad n \rightarrow \infty .
$$

\section{2. Описание модели.}

2.1. Детерминированная модель. В начальный момент времени $t=0$ газ состоит из $n$ частиц, расположенных на прямой в точках с координатами $x_{1}(0), \ldots, x_{n}(0)$. Частицы в рассматриваемой модели являются материальными точками и характеризуются массами $m_{i}$ и координатами $x_{i}(t)$ на оси. Обладая в начальный момент скоростями $v_{i}(0)$, частицы начинают движение под действием гравитационных сил. Сила притяжения здесь пропорциональна произведению масс, т.е. частица массы $\widetilde{m}_{1}$, находящаяся в точке $\widetilde{x}_{1}$, действует на частицу массы $\widetilde{m}_{2}$, находящуюся в точке $\widetilde{x}_{2}$, с силой

$$
F=\gamma \widetilde{m}_{1} \widetilde{m}_{2} \operatorname{sign}\left(\widetilde{x}_{1}-\widetilde{x}_{2}\right),
$$

где $\gamma>0$ - постоянная тяготения. Особо подчеркнем, что сила притяжения не зависит от расстояния, что свойственно одномерным моделям. Сил, отличных от гравитационной, в нашей модели нет.

Итак, система полностью определяется количеством частиц $n$, их массами, координатами и скоростями в начальный момент, а также постоянной $\gamma$. Газ называется холодныл, если все начальные скорости нулевые, и тепльмм в противном случае. Для простоты описания мы предполагаем, что $x_{i}(0)$ упорядочены по возрастанию. 
При описании системы частиц мы придерживаемся правил классической ньютоновской механики. Отсюда ускорение любой частицы пропорционально разности суммарных масс частиц, находяшихся справа и слева от нее.

Одной из ключевых характеристик газа является поведение частиц при столкновениях: частицы слипаются, подчиняясь законам сохранения массы и импульса. Отметим, что при этом энергия системы падает. Такие столкновения называются абсолютно неупругими.

Новые частицы, образовавшиеся в результате слипания, называются кластерами. В дальнейшем сам термин частица мы будем использовать только для обозначения исходных частиц. А кластером будем называть не только результат слипания, но и любую исходную частицу, не успевшую испытать столкновение. Таким образом, газ в любой момент $t>0$ является набором кластеров, каждый из которых состоит из одной или нескольких частиц.

Очевидно, что все частицы рано или поздно слипнутся в один кластер. Тот момент, когда произойдет указанное событие, назовем временем коллапса системы и обозначим $t_{n}^{\text {coll }}$.

В этой работе мы изучаем только те системы, в которых все массы исходных частиц равны между собой, а их сумма есть 1 , т.е. $m_{i}=n^{-1}$, где $1 \leqslant i \leqslant n$. Не умаляя общности, положим $\gamma=1$. Для краткости обозначим $x_{i}=x_{i}(0)$ и $v_{i}=v_{i}(0)$.

2.2. Вероятностная модель. Теперь будем считать, что $x_{i}$ и $v_{i}$ являются случайными величинами, а законы, которым подчиняется поведение системы, остались прежними, детерминистическими. Каждая модель газа, таким образом, полностью характеризуется выбором указанных случайных величин. Задача, которая возникает при таком подходе, - описание в терминах теории вероятностей различных свойств системы в пределе при $n \rightarrow \infty$. Таким образом, мы исследуем характеристики «типичной» системы, состоящей из большого числа частиц.

Начальные положения частиц варьируются в рамках следующих трех моделей, которые на самом деле обладают многими одинаковыми предельными свойствами.

Пуассоновская модель предполагает, что $x_{i}$ есть момент $i$-го скачка пуассоновского процесса интенсивности $n$. Эта модель удобна тем, что для любого $i \geqslant 0$ разности $x_{i+1}-x_{i}$ независимы и распределены экспоненциально, со средним $n^{-1}$. Здесь мы формально полагаем $x_{0}=0$. Для краткости начальные положения частиц в этой модели будем обозначать $X_{i}$.

Во второй модели, именуемой нами независимой равномерно распределенной (н.p.p.) моделью, $x_{i}$ есть $i$-я порядковая статистика выборки из $n$ независимых равномерно распределенных на $[0,1]$ случайных величин. Начальные положения частиц в н.p.p. модели будем обозначать $X_{i}^{\prime}$. 
В рамках решетчатой модели начальные положения частиц фиксированы: $x_{i}=i / n$.

Хотя н.р.p. модель более интересна, но пуассоновская проще из-за уже упомянутой независимости расстояний между частицами. Связь между моделями излагает следуюшая известная лемма.

Лемма 1. Пусть $X_{i}$ - моменть скачков пуассоновского прочесса (произвольной интенсивности). Тогда совместное распределение случайных величин $X_{i}^{\prime}=X_{i} / X_{n+1}$, где $1 \leqslant i \leqslant n$, совпадает с совместиым распределением порядковых статистик выборки из $n$ независимых равномерно распределенных на $[0,1]$ случайных величин.

Начальные скорости $v_{i}$ полагаются независимыми (в совокупности) $с x_{i}$. Кроме того, $v_{i}$ должны быть независимы между собой $u$ одинаково распределены. В холодном газе, как уже говорилось, $v_{i}=0$. В случае теплого газа часто рассматривается нормальное распределение начальных скоростей. Однако мы потребуем лишь $\mathbf{D} v_{1}^{2}=\sigma^{2}<\infty$.

Будем считать, что $\mathbf{E} v_{1}=0$. Общий случай получается из рассматриваемого добавлением к движениям частиц сдвига с постоянной скоростью $\mathbf{E} v_{1}$. При этом все основные характеристики системы, за исключением энергии, остаются прежними.

3. Динамика и кинематика систем. При исследовании систем частиц ключевую роль играет свойство, сформулированное ниже в предложении 1. Оно многократно использовалось в статьях [2]-[7] и поэтому хорошо известно. Для большей общности все формулировки даются для систем с произвольными массами начальных частиц.

В этом разделе число частиц $n$ считается фиксированным.

3.1. Блоки частиц и центры масс. Блоком частиц назовем произвольный набор частиц с идушими подряд номерами. Условимся блок частиц с номерами $i+1, \ldots, i+k$ обозначать $J=(i, i+k]$, сопоставляя ему подмножество отрезка натуральных чисел $[1, n]$. Размером блока естественно называть число его элементов. Заметим, что некоторые частицы блока в какой-то момент могут находиться в одном кластере с частицами, не входящими в блок. Будем говорить, что блок свободен к моменту $t$, если его частицы еще не сталкивались с частицами вне блока. Это определение можно немного ослабить: блок свободен справа (слева) к моменту $t$, если его частицы еше не сталкивались с частицами, находящимися справа (слева) от блока. Введем еще одно обозначение: кластер, состоящий из частиц, входящих в блок $J=(i, i+k]$ (и только из них), будем записывать $K(i, i+k]=K J$.

Очень удобно считать, что исходные частицы не исчезают при столкновениях, а продолжают существовать в составе образовавшихся кластеров. При этом их координаты $x_{i}(t)$ и скорости $v_{i}(t)$ понимаются 
как соответствующие характеристики содержащего их кластера. Таким образом, $x_{i}(t)$ и $v_{i}(t)$ определены при всех $t \geqslant 0$.

Учитывая все сказанное, при $t \geqslant 0$ определим иентр тяжести блока, который будем обозначать

$$
x_{J}(t)=M_{J}^{-1} \sum_{j \in J} m_{j} x_{j}(t), \quad \text { где } \quad M_{J}=\sum_{j \in J} m_{j} .
$$

Аналогично определяется

$$
v_{J}(t)=M_{J}^{-1} \sum_{j \in J} m_{j} v_{j}(t) .
$$

Предложение 1. Пусть блок $J=(i, i+k]$ свободен $\kappa$ моменту $t$. Тогда его чентр тяжести движется как единая частича суммарной массы и суммарного импульса, т.е.

$$
x_{J}(s)=x_{J}(0)+v_{J}(0) s+\frac{\left(M_{J}^{(R)}-M_{J}^{(L)}\right) s^{2}}{2}, \quad 0 \leqslant s \leqslant t,
$$

где $M_{J}^{(R)}=\sum_{j>i+k} m_{j} u M_{J}^{(L)}=\sum_{j \leqslant i} m_{j}-$ массь частии, изначально находящихся соответственно справа и слева от $J$.

Следствие. В холодном газе при любых начальных положениях чаcmuи $t_{n}^{\text {coll }} \leqslant \sqrt{2}$.

Д о к а з а т ел ь с т в о. Очевидно, существует такое $k \in[1, n]$, что блоки $[1, k]$ и $(k, n]$ свободны к моменту $t_{n}^{\text {coll }}$. После несложных преобразований (газ холодный, т.е. $v_{i}=0$ ) получаем $x_{(k, n]}(s)-x_{[1, k]}(s)=$ $x_{(k, n]}(0)-x_{[1, k]}(0)-s^{2} / 2$ для любого $s \leqslant t_{n}^{\text {coll }}$. Поскольку в момент столкновения справедливо $x_{[1, k]}\left(t_{n}^{\text {coll }}\right)=x_{(k, n]}\left(t_{n}^{\text {coll }}\right)$, то $\left(t_{n}^{\text {coll }}\right)^{2} / 2=x_{(k, n]}(0)-$ $x_{[1, k]}(0) \leqslant 1$.

В более общем случае, когда частищы блока могли испытывать столкновения с частицами, находящимися слева (справа) от блока, справедливо следующее предложение.

Предложение 2. Пусть блок $J=(i, i+k]$ свободен справа (слева) $\kappa$ моменту $t$. Тогда

$$
x_{J}(s) \geqslant(\leqslant) x_{J}(0)+v_{J}(0) s+\frac{\left(M_{J}^{(R)}-M_{J}^{(L)}\right) s^{2}}{2}, \quad 0 \leqslant s \leqslant t .
$$

Наконец, введем функцию

$$
x_{J}^{*}(s)=x_{J}(0)+v_{J}(0) s+\frac{\left(M_{J}^{(R)}-M_{J}^{(L)}\right) s^{2}}{2}, \quad s \geqslant 0,
$$

которая описывает воображаемую траекторию движения центра тяжести блока $J$ без учета столкновений со внешними частицами. 
3.2. Время кластеризации. Временем кластеризации $t_{J}^{\mathrm{cl}}$ блока $J$ назовем тот момент, когда все его частицы впервые окажутся в одном кластере (который может содержать частицы не из $J$ ). Время кластеризации $t_{j}^{\mathrm{cl}}$ частицы с номером $j$ - это тот момент, когда она слиплась с $(j+1)$-й частицей. По определению, $t_{j}^{\mathrm{cl}}=t_{[j, j+1]}^{\mathrm{cl}}$. Очевидно, что для любого блока $J=(i, i+k]$ выполняется

$$
t_{J}^{\mathrm{cl}}=\max _{i<j<i+k} t_{j}^{\mathrm{cl}} .
$$

Отсюда понятно наше стремление найти способ вычисления $t_{j}^{\mathrm{cl}}$. Его дает следующее предложение.

Предложение 3. Для любой частииь $1 \leqslant j<n$ время ее кластеризачии есть

$$
t_{j}^{\mathrm{cl}}=\inf \left\{s \geqslant 0: \min _{\substack{0 \leqslant l<j \\ j<k \leqslant n}}\left(x_{(j, k]}^{*}(s)-x_{(l, j]}^{*}(s)\right)=0\right\} .
$$

Несложное доказательство, вытекающее из предложения 2, можно найтии в [4].

3 а м е ч а н и е. Все уравнения (относительно $s$ ) вида $x_{(j, k]}^{*}(s)-$ $x_{(l, j]}^{*}(s)=0$ имеют ровно один неотрицательный корень.

Действительно, из определения величин $x_{J}^{*}(s)$, получаем

$$
x_{(j, k]}^{*}(s)-x_{(l, j]}^{*}(s)=x_{(j, k]}(0)-x_{(l, j]}(0)+\left(v_{(j, k]}(0)-v_{(l, j]}(0)\right) s-\frac{M_{(l, k]} s^{2}}{2} .
$$

Свободный член неотрицателен, а коэффициент при $s^{2}$ меньше нуля.

Теперь мы готовы приступить к изучению стохастических систем частиц.

4. Предел времени кластеризации частицы. Для начала нам потребуется исследовать асимптотику времени кластеризации частиц. До сих пор, когда мы имели дело с подобными величинами, число частиц $n$ было фиксированным и указание на него не требовалось: Теперь же, при переменном $n$, старая запись становится некорректной. Заменим ее на $t_{j, n}^{\mathrm{cl}}$ (время кластеризации $j$-й из $n$ частиц).

При меняющемся $n$ естественно менять и номера частиц. Например, «средняя» частица должна иметь номер $n / 2$. Назовем последовательность (номеров частиц) $r(n)$ типичной, если $\lim r(n)=\lim (n-r(n))=$ $+\infty$ при $n \rightarrow \infty$ и $r(n) \in[1, n]$ для любого $n$.

Лемма 2. В холодном газе для пуассоновской и независимой равномерно распределенной моделей верно следующее. Для любой типичной последовательности $r(n)$ величинь $t_{r(n), n}^{\mathrm{cl}}$ сходятся по распределению $\kappa$ не зависяшему от $r(n)$ пределу. Обозначим

$$
f(t)=\lim _{n \rightarrow \infty} \mathbf{P}\left\{t_{r(n), n}^{\mathrm{cl}}>t\right\}, \quad t \geqslant 0 .
$$


Для $f(t)$ существует явное выражение (8). Эта Функиия непрерывна, монотонно убывает и равна нулю при $t \geqslant 1$.

Более того, если $r(n)<n / 2$ для любого $n$, то при $t \geqslant 0$

$$
\max _{j \in[r(n), n-r(n)]}\left|\mathbf{P}\left\{t_{j, n}^{\mathrm{cl}}>t\right\}-f(t)\right| \longrightarrow 0, \quad n \rightarrow \infty .
$$

3 а м е ч а н и е. Вычислить $f(t)$ автору пока не удалось. Однако компьютерное моделирование показывает, что $f(t)=1-t^{2}$ при $t \in[0,1]$. Столь простой ответ просто обязывает продолжать усилия.

Функцию $f(t)$ будем называть функиией кластеризаиии (холодного газа). Это определение навеяно теоремой 1 из следующего раздела.

Д о к а з а т е л ь с т в о. Часть первая: доказательство для пуассоновской модели. Пусть $n$ фиксировано и $j \in[r(n), n-r(n)]$. Для вычисления $t_{j, n}^{\mathrm{cl}}$ мы воспользуемся формулой (2) из предложения 3 . Так как $m_{i}=n^{-1}$, а газ холодный $\left(v_{i}=0\right)$, то формула (3) примет вид

$$
x_{(j, k]}^{*}(s)-x_{(l, j]}^{*}(s)=\frac{1}{k-j} \sum_{i=j+1}^{k} X_{i}-\frac{1}{j-l} \sum_{i=l+1}^{j} X_{i}-\frac{k-l}{2 n} s^{2} .
$$

Поскольку это выражение монотонно убывает при $s \geqslant 0$, из формулы (2) для любого $t \geqslant 0$ получаем

$$
\left\{t_{j, n}^{\mathrm{cl}}>t\right\} \Longleftrightarrow\left\{\min _{\substack{0 \leqslant l<j \\ j<k \leqslant n}}\left(\frac{1}{k-j} \sum_{i=j+1}^{k} X_{i}-\frac{1}{j-l} \sum_{i=l+1}^{j} X_{i}-\frac{k-l}{2 n} t^{2}\right)>0\right\} .
$$

Преобразуем выражение, соответствующее расстоянию между центрами тяжести:

$$
\begin{aligned}
& \frac{1}{k-j} \sum_{i=j+1}^{k} X_{i}-\frac{1}{j-l} \sum_{i=l+1}^{j} X_{i} \\
& =\frac{1}{k-j} \sum_{i=j+1}^{k}\left(X_{i}-X_{j}\right)+X_{j}-\frac{1}{j-l} \sum_{i=l+1}^{j} X_{i} \\
& =\frac{1}{k-j} \sum_{i=j+1}^{k}\left(X_{i}-X_{j}\right)+\frac{1}{j-l} \sum_{i=l+1}^{j}\left(X_{j}-X_{i}\right) .
\end{aligned}
$$

Ввиду независимости приращений и стационарности пуассоновского процесса, эта случайная величина имеет такое же распределение, как и

$$
\frac{1}{k-j} \sum_{i=1}^{k-j} Z_{i}+\frac{1}{j-l} \sum_{i=1}^{j-l-1} W_{i}
$$


где $Z_{i}$ и $W_{i}$ - моменты скачков независимых пуассоновских процессов интенсивности $n$. Отсюда с учетом формулы (5) для времени кластеризации получаем

$$
\mathbf{P}\left\{t_{j, n}^{\mathrm{cl}}>t\right\}=\mathbf{P}\left\{\min _{\substack{1 \leqslant q \leqslant j \\ 1 \leqslant p \leqslant n-j}}\left(\frac{1}{p} \sum_{i=1}^{p} Z_{i}+\frac{1}{q} \sum_{i=1}^{q-1} W_{i}-\frac{p+q}{2 n} t^{2}\right)>0\right\} .
$$

Завершим преобразования переходом к моментам скачков $P_{i}$ и $Q_{i}$ стандартных независимых пуассоновских процессов:

$$
\mathbf{P}\left\{t_{j, n}^{\mathrm{cl}}>t\right\}=\mathbf{P}\left\{\min _{\substack{1 \leqslant q \leqslant j \\ 1 \leqslant p \leqslant n-j}}\left(\frac{1}{p} \sum_{i=1}^{p} P_{i}+\frac{1}{q} \sum_{i=1}^{q-1} Q_{i}-\frac{p+q}{2} t^{2}\right)>0\right\} .
$$

Поэтому при любом $t$ предел по $n$ выражения $\mathbf{P}\left\{t_{r(n), n}^{\mathrm{cl}}>t\right\}$ существует и не зависит от $r(n)$ (см. определение типичной последовательности). Далее, если $j \in[r(n), n-r(n)]$, то

$$
f(t)<\mathbf{P}\left\{t_{j, n}^{\mathrm{cl}}>t\right\}<\mathbf{P}\left\{\min _{\substack{1 \leqslant q \leqslant r(n) \\ 1 \leqslant p \leqslant r(n)}}\left(\frac{1}{p} \sum_{i=1}^{p} P_{i}+\frac{1}{q} \sum_{i=1}^{q-1} Q_{i}-\frac{p+q}{2} t^{2}\right)>0\right\} .
$$

Отсюда получаем равномерную оценку (4), так как правая часть стремится к $f(t)$ при $n \rightarrow \infty$.

Осталось показать, что $1-f(t)$ является функцией распределения. Это незамедлительно следует из теоремы Прохорова. Действительно, так как $0 \leqslant t_{r(n), n}^{\text {cl }} \leqslant t_{n}^{\text {coll }}$, то, по следствию предложения 1 , распределения случайных величин $t_{r(n), n}^{\mathrm{cl}}$ сосредоточены на $[0, \sqrt{2}]$.

Таким образом, функция кластеризации непрерывна справа. Чтобы получить непрерывность слева, положим $r(n)=n / 3$. Тогда при $n \rightarrow \infty$ правая часть выражения (7) поточечно сходится к $f(t)$, монотонно убывая по $n$. Поскольку сходящиеся функции непрерывны, отсюда следует искомая непрерывность слева для $f(t)$.

Равенство $f(t)=0$ при $t>1$ следует из следующего хорошо известного факта (например, см. [3]): в холодном газе (при любой из трех моделей начальных положений)

$$
t_{n}^{\text {coll }} \stackrel{\mathbf{P}}{\longrightarrow} 1, \quad n \rightarrow \infty
$$

Наконец, для получения более удобного выражения для $f(t)$, в (7) перейдем к пределу по $n$ и воспользуемся свойством непрерывности вероятностной меры:

$$
f(t)=\mathbf{P}\left\{\min _{p \in \mathbf{N}}\left(\frac{1}{p} \sum_{i=1}^{p} P_{i}-\frac{p+1}{2} t^{2}\right)+\min _{q \in \mathbf{N}}\left(\frac{1}{q} \sum_{i=1}^{q-1} Q_{i}-\frac{q-1}{2} t^{2}\right)>0\right\} .
$$


Напомним, что $P_{i}$ и $Q_{i}$ - моменты скачков стандартных независимых пуассоновских процессов. Можно также показать, что

$$
f(t)=e^{t^{2}} \mathbf{P}\left\{\min _{k \in \mathbf{N}} \sum_{i=1}^{k}\left(P_{i}-t^{2} i\right)>0\right\}^{2} .
$$

Часть вторая. Покажем, что н.р.р. модель дает такой же результат, как и пуассоновская. Ограничимся проверкой условия (4).

Преобразуя выражение (5) для того, чтобы явно выделить $t$, положим

$$
G_{j}\left[y_{1}, \ldots, y_{n}\right]=\min _{\substack{1 \leqslant l<j \\ j<k \leqslant n}} \frac{2 n}{k-l}\left(\frac{1}{k-j} \sum_{i=j+1}^{k} y_{i}-\frac{1}{j-l} \sum_{i=l+1}^{j} y_{i}\right)
$$

С использованием этого обозначения (5) примет вид

$$
\left\{t_{j, n}^{\mathrm{cl}}>t\right\} \Longleftrightarrow\left\{G_{j}\left[X_{1}, \ldots, X_{n}\right]>t^{2}\right\}
$$

$\left(t_{j, n}^{\mathrm{cl}}\right.$ здесь, естественно, для пуассоновской модели). В н.р.p. модели выражение для времени кластеризации частицы будет абсолютно таким же, $X_{i}$ лишь заменяется на $X_{i}^{\prime}$. Воспользуемся леммой 1 и осуществим переход от одной модели к другой:

$$
\mathbf{P}\left\{G_{j}\left[X_{1}^{\prime}, \ldots, X_{n}^{\prime}\right]>t^{2}\right\}=\mathbf{P}\left\{G_{j}\left[\frac{X_{1}}{X_{n+1}}, \ldots, \frac{X_{n}}{X_{n+1}}\right]>t^{2}\right\} .
$$

Из определения $G_{j}$ легко видеть, что

$$
G_{j}\left[\frac{X_{1}}{X_{n+1}}, \ldots, \frac{X_{n}}{X_{n+1}}\right]=\frac{G_{j}\left[X_{1}, \ldots, X_{n}\right]}{X_{n+1}} .
$$

Значит, доказывая (4), мы должны установить справедливость равенства

$$
\lim _{n \rightarrow \infty} \max _{j \in[r(n), n-r(n)]}\left|\mathbf{P}\left\{G_{j}\left[X_{1}, \ldots, X_{n}\right]>X_{n+1} t^{2}\right\}-f(t)\right|=0
$$

Для начала заметим, что

$$
X_{n+1} \stackrel{\mathbf{P}}{\longrightarrow} 1
$$

Действительно, переходя к стандартному пуассоновскому процессу, получаем $X_{n+1} \stackrel{\mathrm{d}}{=} P_{n+1} / n$. Но $P_{n+1}$ является суммой $n+1$ независимой экспоненциально распределенной случайной величины со средним 1 , и, следовательно, применим закон больших чисел. 
Для произвольных $\varepsilon \in(0,1 / 2)$ и $j \in[1, n]$ можно вывести

$$
\mathbf{P}\left\{X_{n+1} t^{2}<G_{j}\right\}<\mathbf{P}\left\{(1-\varepsilon) t^{2}<G_{j}\right\}+1-\mathbf{P}\left\{\left|1-X_{n+1}\right|<\varepsilon\right\}
$$

И

$$
\mathbf{P}\left\{X_{n+1} t^{2}<G_{j}\right\}>\mathbf{P}\left\{(1+\varepsilon) t^{2}<G_{j}\right\}-1+\mathbf{P}\left\{\left|1-X_{n+1}\right|<\varepsilon\right\} .
$$

С помощью этих оценок получаем неравенства

$$
\begin{aligned}
\mid \mathbf{P}\{ & \left.X_{n+1} t^{2}<G_{j}\right\}-f(t)|<| \mathbf{P}\left\{(1+\varepsilon) t^{2}<G_{j}\right\}-f(t) \mid \\
& +\left|\mathbf{P}\left\{(1-\varepsilon) t^{2}<G_{j}\right\}-f(t)\right|+1-\mathbf{P}\left\{\left|1-X_{n+1}\right|<\varepsilon\right\} \\
< & \left|\mathbf{P}\left\{(1+\varepsilon) t^{2}<G_{j}\right\}-f\left((1+\varepsilon)^{1 / 2} t\right)\right| \\
& +\left|\mathbf{P}\left\{(1-\varepsilon) t^{2}<G_{j}\right\}-f\left((1-\varepsilon)^{1 / 2} t\right)\right| \\
& +\left\{f\left((1-\varepsilon)^{1 / 2} t\right)-f\left((1+\varepsilon)^{1 / 2} t\right)\right\}+\left\{1-\mathbf{P}\left\{\left|1-X_{n+1}\right|<\varepsilon\right\}\right\},
\end{aligned}
$$

во втором переходе использовалась монотонность $f(t)$. В последнем неравенстве возьмем максимум по $j \in[r(n), n-r(n)]$ и осушествим два предельных перехода, сперва по $n \rightarrow \infty$, а затем по $\varepsilon \rightarrow 0$. При этом мы получим (9). Действительно, максимум суммы не превосходит суммы максимумов. Два первых слагаемых будут равны нулю на основании доказанной первой части, третье - по непрерывности $f(t)$, а четвертое в силу (10). Лемма 2 доказана.

Обратимся теперь к теплому газу. Оказывается, типичным явлением будет мгновенное слипание произвольной частицы с соседней. Более того, мы увидим, что типично мгновенное образование сколь угодно больших кластеров.

Лемма 3. В теплом газе (при любой из рассматриваемьхх моделей начальных положений) для любой типичной последовательности $r(n)$ выполняется

$$
t_{r(n), n}^{\mathrm{cl}} \stackrel{\mathbf{P}}{\longrightarrow} 0, \quad n \rightarrow \infty .
$$

Более того, если $r(n)<n / 2$ для любого $n$, то при $\tau>0$ справедлива равномерная оченка

$$
\min _{j \in[r(n), n-r(n)]} \mathbf{P}\left\{t_{j, n}^{c l}<\tau\right\} \longrightarrow 1, \quad n \rightarrow \infty .
$$

Д о к а з а т е л ь с т в о. Пусть зафиксировано произвольное $\tau>0$, а $j \in[r(n), n-r(n)]$. Снова воспользуемся условием кластеризации из предложения 3. Формула (3) примет вид

$$
x_{(j, k]}^{*}(s)-x_{(l, j]}^{*}(s)=x_{(j, k]}(0)-x_{(l, j]}(0)+\left(v_{(j, k]}(0)-v_{(l, j]}(0)\right) s-\frac{k-l}{2 n} s^{2} .
$$


Коэффициенты этого квадратного трехчлена обозначим $a_{l, k}, b_{l, k}$ и $c_{l, k}$, начиная со старшей степени. Сам трехчлен обозначим $h_{l, k}(s)$. При этом условие кластеризации (2) запишется как

$$
t_{j, n}^{\mathrm{cl}}=\inf \left\{s \geqslant 0: \min _{\substack{0 \leq l<j \\ j<k \leqslant n}} h_{l, k}(s)=0\right\} .
$$

Изучим подробнее уравнения $h_{m, m}(s)=a_{m, m} s^{2}+b_{m, m} s+c_{m, m}=0$. Для них $h_{m, m}(0)=c_{m, m} \geqslant 0$ и $h_{m, m}\left(-c_{m, m} / b_{m, m}\right)=a_{m, m} \cdot\left(c_{m, m} / b_{m, m}\right)^{2}<0$. Поэтому если $b_{m, m}<0$ для какого-то $m$, то неотрицательный корень будет меньше, чем $-c_{m, m} / b_{m, m}$. Значит,

$$
\left\{b_{m, m}<0\right\} \Longrightarrow\left\{t_{j, n}^{\mathrm{cl}}<-\frac{c_{m, m}}{b_{m, m}}\right\} .
$$

Выясним, насколько вероятно найти такое $m$, что $b_{m, m}<0$. Положим $\widetilde{v}_{1}=v_{1}-v_{2}$ и введем новую последовательность центрированных независимых одинаково распределенных случайных величин $\left\{\widetilde{v}_{i}\right\}_{i=1}^{\infty}$. Тогда

$$
m b_{m, m}=\sum_{i=j+1}^{j+m} v_{i}-\sum_{i=j-m+1}^{j} v_{i} \stackrel{\mathrm{d}}{=} \sum_{i=1}^{m} \widetilde{v}_{i} .
$$

Зафиксируем произвольное $\varepsilon>0$. Существует такое $N=N(\varepsilon)$, что

$$
\mathbf{P}\left\{\min _{1 \leqslant m \leqslant N} \sum_{i=1}^{m} \widetilde{v}_{i}<-1\right\}>1-\frac{\varepsilon}{2} .
$$

Справедливость этого утверждения вытекает из соотношения

$$
\mathbf{P}\left\{\frac{1}{\sqrt{n \mathbf{D} u_{1}}} \max _{1 \leqslant m \leqslant n} \sum_{i=1}^{m} u_{i}>\alpha\right\} \rightarrow \frac{2}{\sqrt{2 \pi}} \int_{\alpha}^{\infty} e^{-x^{2} / 2} d x, \quad n \rightarrow \infty,
$$

где $\alpha \geqslant 0$, а $u_{i}$ - независимые одинаково распределенные случайные величины такие, что $\mathbf{D} u_{1}<\infty$ и $\mathbf{E} u_{1}=0$ (см. [8, гл. $\left.2, \S 10\right]$ ). Напомним читателю, что у нас $\mathbf{D} \widetilde{v}_{1}=2 \sigma^{2}<\infty$.

Условимся, что $n$ достаточно велико (такое, что $N \leqslant r(n)$ и $r(n)+$ $N \leqslant n)$. Поскольку

$$
\left\{\min _{1 \leqslant m \leqslant N} m b_{m, m}<-1\right\} \Longrightarrow\left\{\min _{1 \leqslant m \leqslant N} b_{m, m}<-\frac{1}{N}\right\},
$$

из (12) и (13) получаем

$$
\mathbf{P}\left\{\min _{1 \leqslant m \leqslant N} b_{m, m}<-\frac{1}{N}\right\}>1-\frac{\varepsilon}{2} .
$$

Теперь изучим коэффициенты $c_{m, m}$. Учитывая, что $x_{(j, k]}(0) \leqslant x_{k}$ и $x_{l+1} \leqslant x_{(l, j]}(0)$, имеем

$$
\max _{1 \leqslant m \leqslant N} c_{m, m}=c_{N, N} \leqslant x_{j+N}-x_{j-N+1} .
$$


Относительно правой части можно сказать, что для любой из трех моделей начальных положений справедливо

$$
\max _{i \in[r(n), n-r(n)]}\left(x_{i+N}-x_{i-N+1}\right) \stackrel{\mathbf{P}}{\longrightarrow} 0, \quad n \rightarrow \infty .
$$

Для н.p.p. модели это вытекает из теоремы Гливенко, которая гарантирует, что

$$
\max _{1 \leqslant i \leqslant n}\left|X_{i}^{\prime}-\frac{i}{n}\right| \stackrel{\mathbf{P}}{\longrightarrow} 0, \quad n \rightarrow \infty .
$$

А для пуассоновской модели справедливость соотношения (16) следует из его справедливости для н.p.p. модели. Достаточно осуществить уже известный переход от одной модели к другой и вспомнить (10).

Из (15) и (16) получаем, что при всех достаточно больших $n$

$$
\mathbf{P}\left\{\max _{1 \leqslant m \leqslant N} c_{m, m}<\frac{\tau}{N}\right\}>1-\frac{\varepsilon}{2} .
$$

Формулы (14) и (18) дают

$$
\mathbf{P}\left\{\exists m \in[1, N]: 0<-\frac{c_{m, m}}{b_{m, m}}<\tau\right\}>1-\varepsilon .
$$

С учетом импликации (11) отсюда следует, что при больших $n$ выполнено $\mathbf{P}\left\{t_{j, n}^{c l}<\tau\right\}>1-\varepsilon$. Равномерность этой оценки по $j \in[r(n), n-r(n)]$ вытекает из равномерности оценки (16) и независимости оценки (14) от $j$.

Следствие. Для любого натурального $M$

$$
\min _{j \in[r(n), n-r(n)]} \mathbf{P}\left\{t_{[j, j+M], n}^{\mathrm{cl}}<\tau\right\} \longrightarrow 1, \quad n \rightarrow \infty .
$$

Д о к а з а т е л ь с т в о. Легко получается из формулы (1).

5. Предельное количество кластеров. Здесь излагается один из основных полученных результатов - формула для количества кластеров в холодном газе.

Теорема 1. В холодном газе для пуассоновской и н.р.p. моделей при любом $t \geqslant 0$

$$
\frac{K_{n}(t)}{n} \stackrel{\mathbf{P}}{\longrightarrow} f(t), \quad n \rightarrow \infty .
$$

Д о к а з а т е л ь с т в. Из-за ограниченности объема статьи мы проведем доказательство лишь для пуассоновской модели. Доказательство для н.p.p. модели абсолютно аналогично второй части доказательства леммы 2.

Заметим, что доказательство можно проводить лишь для $0 \leqslant t<1$, поскольку для остальных $t$ оно получается автоматически. Действительно, если утверждение теоремы уже доказано для всех $t<1$, то при 
$t=1$ его справедливость сразу следует из непрерывности $f(t)$, равенства $f(1)=0$ и монотонного убывания по $t$ величин $K_{n}(t)$. Для $t>1$ оно очевидно тем более.

Итак, зафиксируем произвольное $t<1$. Каждая слипшаяся со своим правым соседом частица уменьшает исходное количество кластеров (т.е. $n$ ) на один. Следовательно, $K_{n}(t)$ есть разница между $n$ и числом слипшихся со своим соседом частиц (к моменту $t$ ). Иначе, это есть число ча́стиц, не слипшихся со своим соседом справа, т.е.

$$
K_{n}(t)=\#\left\{j \in[1, n-1]: t_{j, n}^{\mathrm{cl}}>t\right\} .
$$

Для любого $\delta \in(0,1 / 6)$ последовательность $r(n)=\delta n$ является типичной. Поэтому при доказательстве мы будем пользоваться тем, что известно предельное время кластеризации каждой частицы с номером $j \in[\delta n, n-\delta n]$ (см. лемму 2).

Имеет место такая оценка:

$\#\left\{j \in[\delta n, n-\delta n]: t_{j, n}^{\mathrm{cl}}>t\right\} \leqslant K_{n}(t) \leqslant \#\left\{j \in[\delta n, n-\delta n]: t_{j, n}^{\mathrm{cl}}>t\right\}+2 \delta n$.

Поскольку $\delta$ выбрано так, что $(1-2 \delta)^{-1}<1+3 \delta$, то отсюда

$$
\limsup _{n \rightarrow \infty}\left|\frac{K_{n}(t)}{n}-\frac{\#\left\{j \in[\delta n, n-\delta n]: t_{j, n}^{\mathrm{cl}}>t\right\}}{(1-2 \delta) n}\right| \leqslant 5 \delta .
$$

Теперь для каждой частицы с номером $j \in[\delta n, n-\delta n]$ определим случайную величину $\xi_{j}^{(n)}=\mathbf{1}_{(t,+\infty)}\left(t_{j, n}^{\mathrm{cl}}\right)$. Тогда

$$
\mathbf{P}\left\{\xi_{j}^{(n)}=0\right\}=\mathbf{P}\left\{t_{j, n}^{\mathrm{cl}} \leqslant t\right\} \quad \text { и } \quad \mathbf{P}\left\{\xi_{j}^{(n)}=1\right\}=\mathbf{P}\left\{t_{j, n}^{\mathrm{cl}}>t\right\} .
$$

В терминах этих величин

$$
\#\left\{j \in[\delta n, n-\delta n]: t_{j, n}^{\mathrm{cl}}>t\right\}=\sum_{j=\delta n}^{n-\delta n} \xi_{j}^{(n)} .
$$

Наша задача - показать, что

$$
\limsup _{n \rightarrow \infty} \mathbf{P}\left\{\left|\frac{1}{(1-2 \delta) n}\left(\sum_{j=\delta n}^{n-\delta n} \xi_{j}^{(n)}-\sum_{j=\delta n}^{n-\delta n} \mathbf{P}\left\{t_{j, n}^{\mathrm{cl}}>t\right\}\right)\right|>\delta^{1 / 3}\right\}<10 \delta^{1 / 3}
$$

По лемме $2, \mathbf{P}\left\{t_{j, n}^{\mathrm{cl}}>t\right\} \rightarrow f(t)$ при $n \rightarrow \infty$ равномерно по $j \in[\delta n, n-\delta n]$. Поэтому из (22)

$$
\limsup _{n \rightarrow \infty} \mathbf{P}\left\{\left|\frac{1}{(1-2 \delta) n} \sum_{j=\delta n}^{n-\delta n} \xi_{j}^{(n)}-f(t)\right|>2 \delta^{1 / 3}\right\}<10 \delta^{1 / 3} .
$$

Полученное соотношение, а также формула (20) справедливы при всех $\delta \in\left(0, \frac{1}{6}\right)$. Из них предельным переходом $\delta \rightarrow 0$ получаем (19), что и требовалось. 
Нам осталось «лишь» доказать (22), в чем и состоит вся сложность. Рассмотрим ковариации

$$
\operatorname{cov}\left(\xi_{i}^{(n)}, \xi_{j}^{(n)}\right)=\mathbf{P}\left\{\xi_{i}^{(n)}=1, \xi_{j}^{(n)}=1\right\}-\mathbf{P}\left\{\xi_{i}^{(n)}=1\right\} \mathbf{P}\left\{\xi_{j}^{(n)}=1\right\} .
$$

Отсюда $\operatorname{cov}\left(\xi_{i}^{(n)}, \xi_{j}^{(n)}\right)<1$. Оценим дисперсию суммы величин $\xi_{j}^{(n)}$ :

$$
\begin{aligned}
& \mathbf{D}\left(\sum_{j=\delta n}^{n-\delta n} \xi_{j}^{(n)}\right)=\sum_{i, j=\delta n}^{n-\delta n} \operatorname{cov}\left(\xi_{i}^{(n)}, \xi_{j}^{(n)}\right)=\sum_{k=0}^{n-2 \delta n} \sum_{\substack{|i-j|=k \\
i, j \in[\delta n, n-\delta n]}} \operatorname{cov}\left(\xi_{i}^{(n)}, \xi_{j}^{(n)}\right) \\
& =\sum_{k=0}^{2 \delta n} \sum_{\substack{|i-j|=k \\
i, j \in[\delta n, n-\delta n]}} \operatorname{cov}\left(\xi_{i}^{(n)}, \xi_{j}^{(n)}\right)+\sum_{k=2 \delta n+1}^{n-2 \delta n} \sum_{\substack{|i-j|=k \\
i, j \in[\delta n, n-\delta n]}} \operatorname{cov}\left(\xi_{i}^{(n)}, \xi_{j}^{(n)}\right) \\
& <\sum_{k=0}^{2 \delta n} 2 n+\sum_{k=2 \delta n+1}^{n-2 \delta n} 2 n \max _{\substack{2 \delta n<|i-j| \\
i, j \in\{\delta n, n-\delta n\}}} \operatorname{cov}\left(\xi_{i}^{(n)}, \xi_{j}^{(n)}\right) \\
& <4 \delta n^{2}+2 n^{2} \max _{\substack{2 \delta n<|i-j| \\
i, j \in[\delta n, n-\delta n]}} \operatorname{cov}\left(\xi_{i}^{(n)}, \xi_{j}^{(n)}\right) .
\end{aligned}
$$

Если мы докажем, что

$$
\limsup _{n \rightarrow \infty} \max _{\substack{2 \delta n<<i-j \mid \\ i, j \in[\delta n, n-\delta n]}} \operatorname{cov}\left(\xi_{i}^{(n)}, \xi_{j}^{(n)}\right) \leqslant 0
$$

то из неравенства Чебышева

$$
\begin{gathered}
\limsup _{n \rightarrow \infty} \mathbf{P}\left\{\left|\frac{1}{(1-2 \delta) n}\left(\sum_{j=\delta n}^{n-\delta n} \xi_{j}^{(n)}-\sum_{j=\delta n}^{n-\delta n} \mathbf{P}\left\{t_{j}^{\mathrm{cl}}>t\right\}\right)\right|>\delta^{1 / 3}\right\} \\
\leqslant \limsup _{n \rightarrow \infty} \frac{1}{(1-2 \delta)^{2} n^{2} \delta^{2 / 3}} \mathbf{D}\left(\sum_{j=\delta n}^{n-\delta n} \xi_{j}^{(n)}\right) \leqslant \frac{4 \delta^{1 / 3}}{(1-2 \delta)^{2}}
\end{gathered}
$$

откуда следует искомое (22).

Таким образом, все свелось к доказательству соотношения (23). Покажем, что для любого $\varepsilon \in(0,1)$ существует такое $N(\varepsilon)$, что при всех $n>N(\varepsilon)$ выполняется

$$
i, j \in[\delta n, n-\delta n], \quad 2 \delta n<|i-j| \Longrightarrow \operatorname{cov}\left(\xi_{i}^{(n)}, \xi_{j}^{(n)}\right)<\varepsilon .
$$

Для краткости введем обозначение

$$
C_{m}\left[l, k, y_{1}, \ldots, y_{n}\right]=\frac{1}{k-m} \sum_{i=m+1}^{k} y_{i}-\frac{1}{m-l} \sum_{i=l+1}^{m} y_{i}-\frac{k-l}{2 n} t^{2} .
$$

Ранее было получено (формула (5)), что в холодном газе для пуассоновской модели

$$
\mathbf{P}\left\{\xi_{m}^{(n)}=1\right\}=\mathbf{P}\left\{t_{m, n}^{\mathrm{cl}}>t\right\}=\mathbf{P}\left\{\min _{\substack{0 \leqslant l<m \\ m<k \leqslant n}} C_{m}\left[l, k, X_{1}, \ldots, X_{n}\right]>0\right\} .
$$


Сперва мы докажем, что для любого $\varepsilon \in(0,1)$ существует такое $N(\varepsilon)$, что при всех $n>N(\varepsilon)$ верна импликация

$$
m \in[\delta n, n-\delta n] \Longrightarrow \mathbf{P}\left\{\min _{\substack{\delta n<k-l \\ 0 \leqslant i<m<k \leqslant n}} C_{m}\left[l, k, X_{1}, \ldots, X_{n}\right]>0\right\}>1-\frac{\varepsilon}{3}
$$

и автоматически

$$
\begin{aligned}
& m \in[\delta n, n-\delta n] \\
& \quad \Longrightarrow \mathbf{P}\left\{\min _{\substack{k<l<\delta n \\
0 \leqslant l<m<k \leqslant n}} C_{m}\left[l, k, X_{1}, \ldots, X_{n}\right]>0\right\}<\mathbf{P}\left\{\xi_{m}^{(n)}=1\right\}+\frac{\varepsilon}{3}
\end{aligned}
$$

В самом деле, по теореме Гливенко имеет место соотношение (17), из которого, с учетом (10), вытекает

$$
\max _{1 \leqslant i \leqslant n}\left|X_{i}-\frac{i}{n}\right| \stackrel{\mathbf{P}}{\longrightarrow} 0, \quad n \rightarrow \infty .
$$

Отсюда следует, что для любого $\varepsilon>0$ сушествует такое $N(\varepsilon)$, что

$$
n>N(\varepsilon) \Longrightarrow \mathbf{P}\left\{\max _{1 \leqslant i \leqslant n}\left|X_{i}-\frac{i}{n}\right|<\delta \frac{1-t^{2}}{4}\right\}>1-\frac{\varepsilon}{3} .
$$

Найденное $N(\varepsilon)$ - искомое. Действительно, справедливо

$$
\begin{aligned}
& \max _{1 \leqslant i \leqslant n}\left|X_{i}-\frac{i}{n}\right|<\delta \frac{1-t^{2}}{4} \\
& \Longrightarrow \forall l, k, m \in[1, n] \quad \mid \frac{1}{k-m} \sum_{i=m+1}^{k} X_{i} \\
& \quad-\frac{1}{m-l} \sum_{i=l+1}^{m} X_{i}-\frac{k-l}{2 n} \mid<\delta \frac{1-t^{2}}{2}
\end{aligned}
$$

и поэтому

$$
\begin{aligned}
& \max _{1 \leqslant i \leqslant n}\left|X_{i}-\frac{i}{n}\right|<\delta \frac{1-t^{2}}{4} \\
& \Longrightarrow \forall l, k, m \in[1, n] \quad C_{m}\left[l, k, X_{1}, \ldots, X_{n}\right]>\left(\frac{k-l}{2 n}-\frac{\delta}{2}\right)\left(1-t^{2}\right) .
\end{aligned}
$$

Легко видеть, что отсюда, с учетом условия (27), следует (25).

Теперь, когда получено (25), завершим наши усилия, доказав (23), а точнее, эквивалентное ему $(24)$. В качестве искомого $N(\varepsilon)$ возьмем найденное в (25). Пусть $n>N(\varepsilon)$ и $i, j \in[\delta n, n-\delta n]$ таковы, что $2 \delta n<|i-j|$. Считая без ограничения общности, что $i<j$, получаем

$$
\begin{aligned}
& \mathbf{P}\left\{\xi_{i}^{(n)}=1, \xi_{j}^{(n)}=1\right\} \\
& \quad=\mathbf{P}\left\{\min _{\substack{0 \leqslant l<i \\
i<k \leqslant n}} C_{i}\left[l, k, X_{1}, \ldots, X_{n}\right]>0, \min _{\substack{0 \leqslant p<j \\
j<q \leqslant n}} C_{j}\left[p, q, X_{1}, \ldots, X_{n}\right]>0\right\}
\end{aligned}
$$




$$
\begin{aligned}
< & \mathbf{P}\left\{\min _{\substack{k-l \leqslant \delta n \\
0 \leqslant l<i<k \leqslant n}} C_{i}\left[l, k, X_{1}, \ldots, X_{n}\right]>0, \min _{\substack{q-p \leqslant \delta n \\
0 \leqslant p<j<q \leqslant n}} C_{j}\left[p, q, X_{1}, \ldots, X_{n}\right]>0\right\} \\
= & \mathbf{P}\left\{\min _{\substack{k<l<\delta n \\
0 \leqslant l<i<k \leqslant n}} C_{i}\left[l, k, X_{1}, \ldots, X_{n}\right]>0\right\} \\
& \times \mathbf{P}\left\{\min _{\substack{q-p \leqslant \delta n \\
0 \leqslant p<j<q \leqslant n}} C_{j}\left[p, q, X_{1}, \ldots, X_{n}\right]>0\right\} .
\end{aligned}
$$

Объясним последний переход. Первый минимум есть функция от $X_{1}, \ldots, X_{i+\delta n-1}$. Согласно преобразованиям (6),

$$
\begin{aligned}
& C_{j}\left[p, q, X_{1}, \ldots, X_{n}\right] \\
& \quad=C_{j}\left[p, q, X_{j}-X_{1}, \ldots, X_{j}-X_{j-1}, 0, X_{j+1}-X_{j}, \ldots, X_{n}-X_{j}\right] .
\end{aligned}
$$

Поэтому второй минимум есть функция от $X_{j}-X_{j-\delta n+1}, \ldots, X_{j}-$ $X_{j-1}, 0, X_{j+1}-X_{j}, \ldots, X_{n}-X_{j}$. Остается лишь вспомнить, что $2 \delta n<$ $|i-j|$, и воспользоваться независимостью приращений пуассоновского процесса.

Теперь, используя (26), немедленно получаем

$$
\mathbf{P}\left\{\xi_{i}^{(n)}=1, \xi_{j}^{(n)}=1\right\}<\left(\mathbf{P}\left\{\xi_{i}^{(n)}=1\right\}+\frac{\varepsilon}{3}\right)\left(\mathbf{P}\left\{\xi_{j}^{(n)}=1\right\}+\frac{\varepsilon}{3}\right) .
$$

Значит, $\operatorname{cov}\left(\xi_{i}^{(n)}, \xi_{j}^{(n)}\right)<2 \varepsilon / 3+\varepsilon^{2} / 9<\varepsilon$, это и есть (24). Теорема 1 доказана.

Теперь попытаемся сформулировать аналогичное утверждение для теплого газа. Следующая лемма не дает точной оценки количества кластеров. Однако она будет чрезвычайно полезна в следуюшем разделе.

Зафиксируем произвольное натуральное $M$, а также $\tau>0$. Обозначим

$$
\mathscr{K}_{n}^{M, \tau}=\left\{k \in\left[1, \frac{n}{M}\right]: t_{((k-1) M, k M], n}^{\mathrm{cl}}<\tau\right\} .
$$

Эта величина описывает количество кластеризовавшихся к моменту $\tau$ блоков вида $((k-1) M, k M]$.

Лемма 4. В теплом газе (при любой из трех моделей начальных положений) для произвольных $M \in \mathbf{N} u \tau>0$ справедливо

$$
\frac{\# \mathscr{K}_{n}^{M, \tau}}{n / M} \stackrel{\mathbf{P}}{\longrightarrow} 1, \quad n \rightarrow \infty .
$$

Д о к а з а т е л ь с т в о. Оно идентично доказательству теоремы 1 и самостоятельного интереса не представляет. Там мы воспользовались леммой 2 , а здесь, соответственно, потребуется следствие леммы 3.

Итак, рассмотрим произвольное $\delta \in(0,1 / 6)$. Аналогично определению $(21)$, для каждого $k \in[\delta n / M,(1-\delta) n / M]$ положим $\xi_{k}^{(n)}=$ $\mathbf{1}_{[0, \tau)}\left(t_{((k-1) M, k M], n}^{\mathrm{cl}}\right)$. Как и ранее, достаточно доказать аналог $(24)$, т.е. 
проверить, что для любого $\varepsilon \in(0,1)$ существует такое $N(\varepsilon)$, что при всех $n>N(\varepsilon)$ выполняется

$$
i, j \in\left[\frac{\delta n}{M},(1-\delta) \frac{n}{M}\right], \quad \frac{2 \delta n}{M}<|i-j| \Longrightarrow \operatorname{cov}\left(\xi_{i}^{(n)}, \xi_{j}^{(n)}\right)<\varepsilon .
$$

В отличие от предыдущего доказательства, проверка не составит труда. По следствию леммы 3 , существует $N(\varepsilon)$ такое, что если $n>N(\varepsilon)$, то

$$
j \in[\delta n,(1-\delta) n] \Longrightarrow \mathbf{P}\left\{t_{[j, j+M], n}^{\mathrm{cl}}<\tau\right\}>1-\frac{\varepsilon}{2}
$$

Следовательно, при $n>N(\varepsilon)$

$$
k \in\left[\frac{\delta n}{M},(1-\delta) \frac{n}{M}\right] \Longrightarrow \mathbf{P}\left\{\xi_{k}^{(n)}=1\right\}>1-\frac{\varepsilon}{2} .
$$

Поэтому, если $n>N(\varepsilon)$ и $i, j \in[\delta n / M,(1-\delta) n / M]$, то

$$
\operatorname{cov}\left(\xi_{i}^{(n)}, \xi_{j}^{(n)}\right)<1-\mathbf{P}\left\{\xi_{i}^{(n)}=1\right\} \mathbf{P}\left\{\xi_{j}^{(n)}=1\right\}<1-\left(1-\frac{\varepsilon}{2}\right)^{2}<\varepsilon .
$$

Положив $M=1$, получаем такое следствие.

Следствие. $B$ теплом газе для любого $t>0$ справедливо

$$
\frac{K_{n}(t)}{n} \stackrel{\mathbf{P}}{\longrightarrow} 0, \quad n \rightarrow \infty .
$$

6. Предельная энергия. Основная теорема этого раздела описывает мгновенное охлаждение теплого газа. Кроме того, здесь дается явная формула для энергии.

Поясним полученные результаты. Изменение энергии холодного газа происходит по следующим причинам. С одной стороны, абсолютные скорости подавляющего большинства частиц возрастают из-за гравитации, и в силу этого энергия системы увеличивается. С другой стороны, при столкновениях частиц энергия падает. В теореме 3 , в частности, показано, что в холодном газе процессом уменьшения энергии при столкновениях можно пренебречь. В случае теплого газа происходит мгновенное образование больших кластеров, за счет чего начальные скорости усредняются и практически исчезают. После этого они меняются так же, как и в холодном газе, т.е. опять можно не учитывать уменьшение энергии из-за столкновений.

Теорема 2. Тепльй газ (при любой из трех моделей начальных положений) мәновенно охлаждается: в начальный момент времени

$$
E_{n}(0) \stackrel{\mathbf{P}}{\longrightarrow} \frac{\sigma^{2}}{2}, \quad n \rightarrow \infty,
$$

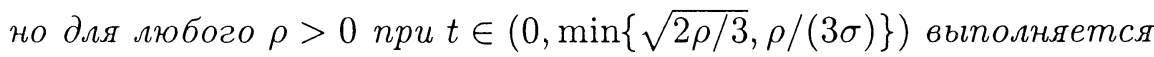

$$
\lim _{n \rightarrow \infty} \mathbf{P}\left\{E_{n}(t)<\rho\right\}=1
$$


Д ок а з а т ел ь с т о. Первая часть теоремы немедленно следует из закона больших чисел.

Для оценки энергии системы в произвольный момент времени $t \in(0, \min \{\sqrt{2 \rho / 3}, \rho /(3 \sigma)\})$ представим множество $[1, n]$ в виде $[1, n]=$ $\bigcup_{j=1}^{m} J_{j}$, где $J_{j}$ - блоки, соответствующие существуюшим в момент $t$ кластерам.

Рассмотрим произвольный кластер $K=K J_{j}$, состоящий из $k=\# J_{j}$ исходных частиц. Так как все абсолютные ускорения $\left|a_{i}\right|$ меньше 1 , то $\left|v_{K}(t)\right|<k^{-1}\left|\sum_{i \in J_{j}} v_{i}\right|+t$. Отсюда для энергии кластера $K$

$$
E_{K}(t)<\frac{k}{2 n}\left(k^{-1}\left|\sum_{i \in J_{j}} v_{i}\right|+t\right)^{2} \leqslant \frac{1}{2 n k}\left(\sum_{i \in J_{j}} v_{i}\right)^{2}+\frac{t}{n} \sum_{i \in J_{j}}\left|v_{i}\right|+\frac{k t^{2}}{2 n} .
$$

Значит, для энергии всей системы

$$
E_{n}(t)<\frac{1}{2 n} \sum_{j=1}^{m} \# J_{j}^{-1}\left(\sum_{i \in J_{j}} v_{i}\right)^{2}+\frac{t}{n} \sum_{i=1}^{n}\left|v_{i}\right|+\frac{t^{2}}{2} .
$$

Разберемся с каждым из трех слагаемых по отдельности. Для третьего слагаемого, которое не является случайным,

$$
\lim _{n \rightarrow \infty} \mathbf{P}\left\{\frac{t^{2}}{2}<\frac{\rho}{3}\right\}=1 .
$$

Напоминаем, что $t$ было выбрано достаточно маленьким. Далее; для второго слагаемого, используя закон больших чисел и то, что $\mathbf{E}\left|v_{1}\right| \leq \sigma$, получаем

$$
\lim _{n \rightarrow \infty} \mathbf{P}\left\{\frac{t}{n} \sum_{i=1}^{n}\left|v_{i}\right|<\frac{\rho}{3}\right\}=\lim _{n \rightarrow \infty} \mathbf{P}\left\{\frac{1}{n} \sum_{i=1}^{n}\left|v_{i}\right|<\frac{\rho}{3 t}\right\}=1 .
$$

Таким образом, мы оценили возможное увеличение энергии изза гравитации. Оставшееся слагаемое является функцией от начальных скоростей. Для его оценки воспользуемся неравенством КошиБуняковского.

Пусть $M \in \mathbf{N}$. Снова рассмотрим произвольный кластер $K=K J_{j}$. Максимальное по включению множество вида $(k M,(k+i) M]$, содержащееся в $J_{j}$, обозначим $I^{M}$; возможно, это множество пусто. Теперь $J_{j}=I^{M} \cup I$, где $I-$ дополнение $I^{M}$ до $J_{j}$. Далее, разобьем $I^{M}$ на непересекаюшиеся подмножества $I_{1}, \ldots, I_{s}$, каждое из которых есть отрезок вида $((k-1) M, k M]$. Естественно, что для таких $k$ справедливо $k \in \mathscr{K}:=\mathscr{K}_{n}^{M, t} ;$ напоминаем, что определение множества $\mathscr{K}_{n}^{M, t}$ было дано в (28). Таким образом, $J_{j}=\left(\bigcup_{\ell=1}^{s} I_{\ell}\right) \cup I$.

В этих обозначениях из неравенства Коши-Буняковского получаем

$$
\# J_{j}^{-1}\left(\sum_{i \in J_{j}} v_{i}\right)^{2} \leqslant \sum_{\ell=1}^{s} \frac{1}{M}\left(\sum_{i \in I_{\ell}} v_{i}\right)^{2}+\sum_{i \in I} v_{i}^{2} .
$$


Отсюда для первого слагаемого суммы (29)

$$
\begin{aligned}
\frac{1}{2 n} \sum_{j=1}^{m} \# J_{j}^{-1}\left(\sum_{i \in J_{j}} v_{i}\right)^{2} \leqslant & \frac{1}{2 n} \sum_{k \in \mathscr{K}} \frac{1}{M}\left(\sum_{i=(k-1) M+1}^{k M} v_{i}\right)^{2} \\
& +\frac{1}{2 n} \sum_{k \notin \mathscr{K}} \sum_{i=(k-1)}^{k M} v_{i}^{2}
\end{aligned}
$$

По лемме 4 , для любых $\delta, \varepsilon>0$ существует такое $N_{1}(\varepsilon)$, что при Bcex $n>N_{1}(\varepsilon)$

$$
\mathbf{P}\left\{\frac{\# \mathscr{K}}{n / M}>1-\delta\right\}>1-\frac{\varepsilon}{2} .
$$

Значит, с вероятностью, превосходящей $1-\varepsilon / 2$, во втором слагаемом суммы (32) суммируется менее чем $\delta n$ величин $v_{i}^{2}$. Поэтому

$$
\mathbf{P}\left\{\frac{1}{2 n} \sum_{k \notin \mathscr{K}} \sum_{i=(k-1) M+1}^{k M} v_{i}^{2}<\frac{1}{2 n} \sum_{i=(1-\delta) n}^{n}\left(v^{2}\right)_{i: n}\right\}>1-\frac{\varepsilon}{2}
$$

$\left(\right.$ где $\left(v^{2}\right)_{i: n}$ - порядковые статистики для серий величин $\left.\left\{v_{i}^{2}\right\}_{i=1}^{n}\right)$.

Обозначим $H_{\delta}$ такой симметричный относительно 0 отрезок, что $P_{v}\left(H_{\delta}\right)=1-\delta$, где $P_{v}$ - мера, являющаяся распределением скоростей. Используя теорему Гливенко и закон больших чисел, получаем

$$
\frac{1}{2 n} \sum_{i=(1-\delta) n}^{n}\left(v^{2}\right)_{i: n} \stackrel{\mathbf{P}}{\longrightarrow} \frac{1}{2} \int_{\mathbf{R} \backslash H_{\delta}} x^{2} d P_{v}(x) .
$$

По абсолютной непрерывности интеграла (второй момент скоростей существует) выражение в правой части стремится к 0 при $\delta \rightarrow 0$. Поэтому при всех достаточно малых $\delta>0$ для произвольного $\varepsilon>0$ сушествует такое $N_{2}(\varepsilon)$, что при всех $n>N_{2}(\varepsilon)$

$$
\mathbf{P}\left\{\frac{1}{2 n} \sum_{i=(1-\delta) n}^{n}\left(v^{2}\right)_{i: n}<\frac{\rho}{6}\right\}>1-\frac{\varepsilon}{2} .
$$

Отсюда и из (33) следует, что при всех малых $\delta>0$ для любого $\varepsilon>0$ и всех $n>\max \left\{N_{1}(\varepsilon), N_{2}(\varepsilon)\right\}$

$$
\mathbf{P}\left\{\frac{1}{2 n} \sum_{k \notin \mathscr{K}} \sum_{i=(k-1)}^{k M} v_{i}^{2}<\frac{\rho}{6}\right\}>1-\varepsilon .
$$

Значит,

$$
\lim _{n \rightarrow \infty} \mathbf{P}\left\{\frac{1}{2 n} \sum_{k \notin \mathscr{K}} \sum_{i=(k-1) M+1}^{k M} v_{i}^{2}<\frac{\rho}{6}\right\}=1
$$


Осталось разобраться лишь с первым слагаемым в правой части (32). Оно, очевидно, мажорируется полной суммой:

$$
\begin{aligned}
\frac{1}{2 n} \sum_{k \in \mathscr{K}} \frac{1}{M}\left(\sum_{i=(k-1) M+1}^{k M} v_{i}\right)^{2} & \leqslant \frac{1}{2 n} \sum_{k=1}^{n / M} \frac{1}{M}\left(\sum_{i=(k-1) M+1}^{k M} v_{i}\right)^{2} \\
& =\frac{M}{2 n} \sum_{k=1}^{n / M}\left(\frac{1}{M} \sum_{i=(k-1) M+1}^{k M} v_{i}\right)^{2}
\end{aligned}
$$

Определим новые серии случайных величин:

$$
\eta_{k}^{(n)}:=\left(\frac{1}{M} \sum_{i=(k-1) M+1}^{k M} v_{i}\right)^{2}, \quad k \in\left[1, \frac{n}{M}\right] .
$$

В каждой серии эти величины независимы, одинаково распределены и, как легко убедиться, имеют математические ожидания, равные $\sigma^{2} M^{-1}$. Вспомним теперь про то, что $M$ было произвольным, и выберем его так, чтобы $\sigma^{2} M^{-1}<\rho / 6$. Тогда по закону больших чисел

$$
\lim _{n \rightarrow \infty} \mathbf{P}\left\{\frac{M}{2 n} \sum_{k=1}^{n / M} \eta_{k}<\frac{\rho}{6}\right\} \geqslant \lim _{n \rightarrow \infty} \mathbf{P}\left\{\left|\frac{M}{n} \sum_{k=1}^{n / M} \eta_{k}-\frac{\sigma^{2}}{M}\right|<\frac{\rho}{6}\right\}=1 .
$$

Учитывая (30), (31), (34) и (35), получаем искомое. Теорема 2 доказана.

Теперь мы можем приступить к доказательству формул для предельной энергии. Но сперва обозначим $L_{n}(t)$ размер максимального клаcmера к моменту $t$. В холодном газе для пуассоновской и н.р.р. моделей при $t<1$ имеем $L_{n}(t) \sim \ln n$ по вероятности (см. [3] и [4]). Отсюда во всех трех моделях

$$
\frac{L_{n}(t)}{n} \stackrel{\mathbf{P}}{\longrightarrow} 0, \quad n \rightarrow \infty
$$

(для решетчатой модели $L_{n}(t)=1$ при $t<1$ ).

Теорема 3. В холодном газе (при любой из трех моделей начальньх положений) для любого $t \in[0,1)$ справедливо

$$
E_{n}(t) \stackrel{\mathbf{P}}{\longrightarrow} \frac{t^{2}}{6}, \quad n \rightarrow \infty \text {. }
$$

Д ок аз а т ель с т в о. Зафиксируем произвольное $t \in[0,1)$. Предположим, что к моменту $t$ образовался кластер $K=K(l, l+k]$. В начальный момент времени произвольная частица с номером $i \in(l, l+k]$ испытывает ускорение $a_{i}(0)=(n-2 i+1) / n$ (с вероятностью 1$)$. Так как блок $(l, l+k]$ свободен к моменту $t$, а все его частицы уже входят в кластер $K$, то справедливо $x_{(l, l+k]}(t)=x_{K}(t)=x_{i}(t)$ и $v_{(l, l+k]}(t)=v_{K}(t)=$ $v_{i}(t)$. Воспользуемся предложением 1 и получим $v_{i}(t)=((n-2 l-k) / n) t$. 
Значит, $\left|v_{i}(t)-a_{i}(0) t\right|<k t / n$; учитывая $\left|a_{i}(0)\right|<1$ и $k \leqslant n$, из формулы разности квадратов выводим неравенство

$$
\left|v_{i}(t)^{2}-a_{i}(0)^{2} t^{2}\right|<2 \frac{k}{n} t^{2}
$$

Учитывая (36), получаем

$$
\sup _{i \in[1, n]}\left|v_{i}(t)^{2}-a_{i}(0)^{2} t^{2}\right| \stackrel{\mathbf{P}}{\longrightarrow} 0, \quad n \rightarrow \infty
$$

и отсюда

$$
E_{n}(t)-\frac{1}{2 n} \sum_{i=1}^{n}\left(\frac{n-2 i+1}{n} t\right)^{2} \stackrel{\mathbf{P}}{\longrightarrow} 0, \quad n \rightarrow \infty .
$$

Вычисление суммы завершает доказательство.

Обратимся теперь к теплому газу. В доказательстве следующей теоремы мы снова воспользуемся идеей об оценке скорости произвольной частицы при помощи размера максимального кластера. Однако для этого потребуется наложить более жесткие условия на $v_{i}$. Согласно [4], при $t<1$ для всех трех моделей начальных положений $L_{n}(t) \sim n^{2 / 3} \ln n^{1 / 3}$ по вероятности, но для этого нужно существование 7-х моментов у скоростей. Нам же требуется лишь $L_{n}(t)=o(n)$. Для решетчатой модели начальных положений этот результат присутствует в [6], при более слабом условии существования моментов порядка $4+\varepsilon$.

Теорема 4. В теплом газе (для любой из трех моделей начальных положений) справедливо

$$
E_{n}(0) \stackrel{\mathbf{P}}{\longrightarrow} \frac{\sigma^{2}}{2}, \quad n \rightarrow \infty .
$$

Если для произвольного $t \in(0,1)$ выполняется условие $(36)$, то

$$
E_{n}(t) \stackrel{\mathbf{P}}{\longrightarrow} \frac{t^{2}}{6}, \quad n \rightarrow \infty
$$

В частности, если $\mathbf{E}\left|v_{1}\right|^{c}<\infty(c=7$ для пуассоновской и н.р.p. моделей, с > 4 для решетчатой), то (37) выполняется при всех $t \in(0,1)$.

Д о к а з а т е л ь с т в о. Снова зафиксируем произвольное $t \in(0,1)$ и $\tau \in(0, t)$. Предположим, что к моменту $t$ образовался кластер $K=K(l, l+k]$. Опять воспользуемся предложением 1 :

$$
v_{i}(t)=\frac{1}{k} \sum_{j=l+1}^{l+k} v_{j}(\tau)+\frac{n-2 l-k}{n}(t-\tau) .
$$

Отсюда, аналогично предыдущему доказательству,

$$
\left|v_{i}(t)-a_{i}(0)(t-\tau)\right|<\left|\frac{1}{k} \sum_{j=l+1}^{l+k} v_{j}(\tau)\right|+\frac{k}{n}(t-\tau)<\left|\frac{1}{k} \sum_{j=l+1}^{l+k} v_{j}(\tau)\right|+\frac{k}{n} t
$$


учитывая, что $k \leqslant n$, получаем

$$
\left|v_{i}(t)^{2}-a_{i}(0)^{2}(t-\tau)^{2}\right|<\left(\left|\frac{1}{k} \sum_{j=l+1}^{l+k} v_{j}(\tau)\right|+\frac{k}{n} t\right)\left(\left|\frac{1}{k} \sum_{j=l+1}^{l+k} v_{j}(\tau)\right|+2 t\right) .
$$

Раскрыв скобки, приходим к неравенству

$$
\left|v_{i}(t)^{2}-a_{i}(0)^{2}(t-\tau)^{2}\right|<\left(\frac{1}{k} \sum_{j=l+1}^{l+k} v_{j}(\tau)\right)^{2}+\frac{3}{k} t\left|\sum_{j=l+1}^{l+k} v_{j}(\tau)\right|+2 \frac{k}{n} t^{2}
$$

Значит, для энергии кластера $K=K(l, l+k]$

$$
\begin{aligned}
\left|E_{K}(t)-\frac{1}{2 n} \sum_{i=l+1}^{l+k} a_{i}(0)^{2}(t-\tau)^{2}\right|< & \frac{k}{2 n}\left(\frac{1}{k} \sum_{i=l+1}^{l+k} v_{i}(\tau)\right)^{2} \\
& +\frac{3}{2 n} t\left|\sum_{i=l+1}^{l+k} v_{i}(\tau)\right|+\frac{k^{2}}{n^{2}} t^{2} .
\end{aligned}
$$

Оценив второе слагаемое суммой модулей, а первое при помощи неравенства Коши-Буняковского, получаем

$\left|E_{K}(t)-\frac{1}{2 n} \sum_{i=l+1}^{l+k} a_{i}(0)^{2}(t-\tau)^{2}\right|<\frac{1}{2 n} \sum_{i=l+1}^{l+k} v_{i}(\tau)^{2}+\frac{3}{2 n} t \sum_{i=l+1}^{l+k}\left|v_{i}(\tau)\right|+\frac{k^{2}}{n^{2}} t^{2}$.

Поскольку $k^{2} \leqslant L_{n}(t) k$, а суммарное количество частиц во всех кластерах равно $n$,

$$
\begin{aligned}
& \left|E_{n}(t)-\frac{1}{2 n} \sum_{i=1}^{n} a_{i}(0)^{2}(t-\tau)^{2}\right| \\
& \quad<\frac{1}{2 n} \sum_{i=1}^{n} v_{i}(\tau)^{2}+\frac{3}{2 n} t \sum_{i=1}^{n}\left|v_{i}(\tau)\right|+\frac{L_{n}(t)}{n} t^{2} \\
& \quad \leqslant \frac{1}{2 n} \sum_{i=1}^{n} v_{i}(\tau)^{2}+\frac{3}{2} t\left(\frac{1}{n} \sum_{i=1}^{n} v_{i}(\tau)^{2}\right)^{1 / 2}+\frac{L_{n}(t)}{n} t^{2} \\
& \quad=E_{n}(\tau)+\frac{3}{2} t \sqrt{2 E_{n}(\tau)}+\frac{L_{n}(t)}{n} t^{2},
\end{aligned}
$$

для второй оценки мы снова использовали неравенство КошиБуняковского. Теперь зафиксируем произвольное $\rho>0$. По теореме 2 и условию (36), из (38) имеем

$$
\lim _{n \rightarrow \infty} \mathbf{P}\left\{\left|E_{n}(t)-\frac{1}{2 n} \sum_{i=1}^{n} a_{i}(0)^{2}(t-\tau)^{2}\right|<\rho\right\}=1
$$

для всех достаточно малых $\tau$. На этом доказательство завершается, ведь при всех малых $\tau$ и больших $n$ справедливо

$$
\mathbf{P}\left\{\left|E_{n}(t)-\frac{t^{2}}{6}\right|<2 \rho\right\} \geqslant \mathbf{P}\left\{\left|E_{n}(t)-\frac{1}{2 n} \sum_{i=1}^{n} a_{i}(0)^{2}(t-\tau)^{2}\right|<\rho\right\} .
$$


7. Компьютерное моделирование. Теорема 1 , являющаяся одним из основных результатов статьи, была обнаружена эмпирически. В результате многочисленных запусков программы, реализующей рассматриваемую модель холодного гравитационного газа, автор обнаружил, что график функции $K_{n}(t) / n$ при больших $n$ сильно напоминает параболу $1-t^{2}$, где $t \in[0,1]$. Это соответствует соотношению (19). Как уже говорилось, равенство $f(t)=1-t^{2}$ пока доказать не удалось, однако проведенное компьютерное моделирование случайной величины из выражения (8) недвусмысленно указывает на его справедливость.

При моделировании была реализована н.р.р. модель начальных положений. В случае теплого газа начальные скорости брались нормальными с параметрами 0 и 1.

На рис. 1 представлены графики функций $K_{n}(t)$ при $n=100$ и $n=1000$ соответственно. Каждый из них получен в результате однократного запуска моделируюшей программы. При $n=5000$ отклонение от предельной функции оказалось настолько малым, что было абсолютно незаметно при выбранном размере рисунка.
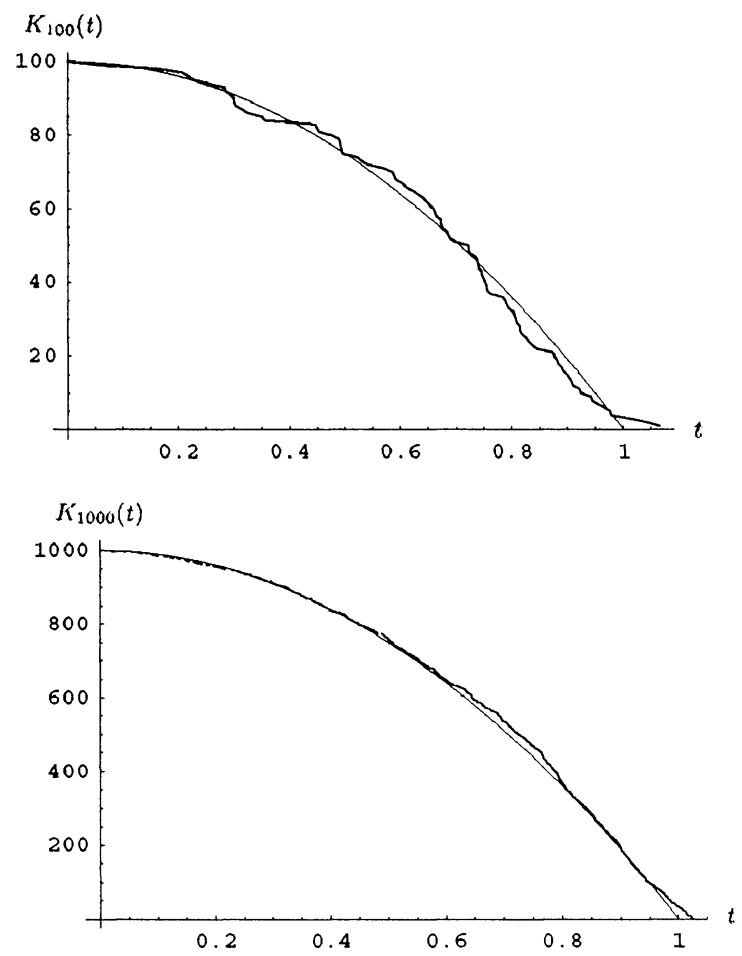

Рис. 1. $K_{100}(t)$ и $K_{1000}(t)$ в холодном газе.

При изучении энергии оказалось, что в холодном газе достаточно хорошее приближение к предельной функции (см. теорему 3 ) достигается 
уже при $n=100$. Это подтверждает рис. 2 .

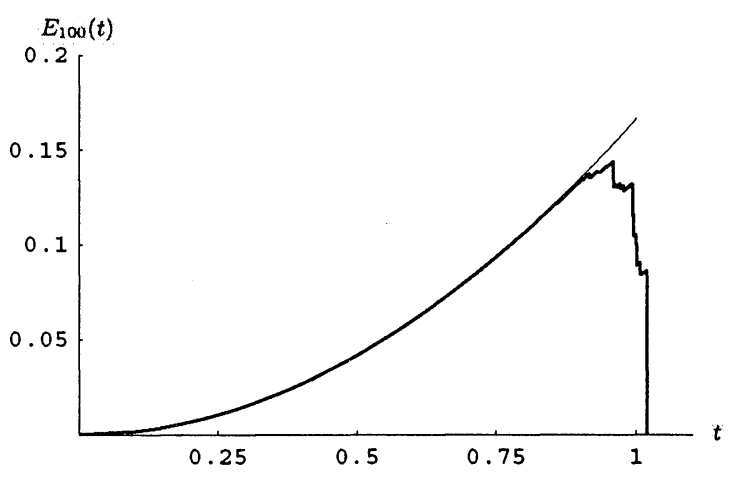

Рис. 2. $E_{100}(t)$ в холодном газе.

В теплом газе сходимость энергии к пределу (см. теорему 4) происходит гораздо медленнее. На рис. 3 изображены графики функций $E_{n}(t)$ при $n=500$ и $n=5000$.
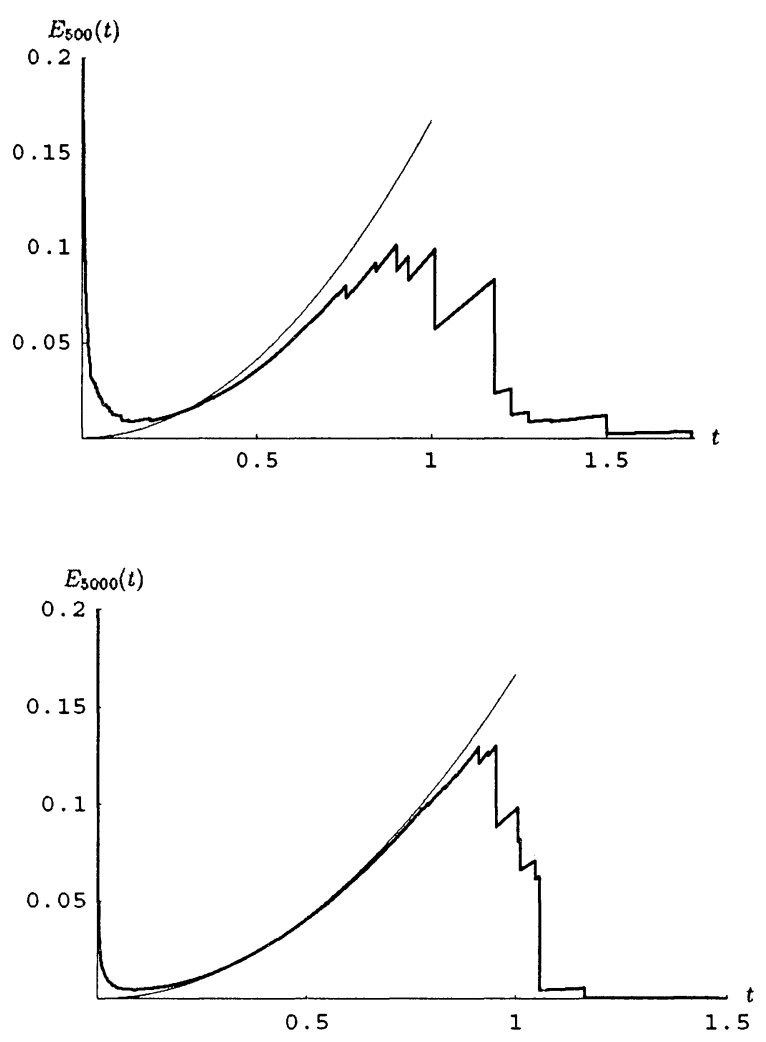

Рис. 3. $E_{500}(t)$ и $E_{5000}(t)$ в теплом газе. 
Отклонение от предельной функции при $t$, близких к 1 , достаточно велико даже при $n=10000$. Однако рис. 3 убедительно свидетельствует об описываемом теоремой 2 мгновенном охлаждении теплого газа.

Автор выражает глубокую признательность своему руководителю М. А. Лифшицу, чьи замечания позволили значительно улучшить изложение результатов и исправить ряд неточностей.

\section{СПИСОК ЛИТЕРАТУРЫ}

1. Shandarin S.F., Zel'dovich Ya.B. The large-scale structure of the universe: turbulence, intermittency, structures in a self-gravitating medium. - Rev. Modern Phys., 1989 , v. 61 , № 2 , p. $185-220$.

2. Martin Ph. A., Piasecki J. Aggregation dynamics in a self-gravitating one-dimensional gas. - J. Statist. Phys., 1996, v. 84, № 3-4, p. 837-857.

3. Giraud C. Clustering in a self-gravitating one-dimensional gas at zero temperature. J. Statist. Phys., 2001, v. 105, № 3-4, p. 585-604.

4. Lifshits M., Shi $Z$. Aggregation rates in one-dimensional stochastic systems with adhesion and gravitation. - Ann. Probab., 2005, v. 33, p. 53-81.

5. Bonvin J. C., Martin Ph. A., Piasecki J., Zotos X. Statistics of mass aggregation in a self-gravitating one-dimensional gas. - J. Statist. Phys., 1998, v. 91, № 1-2, p. 177197.

6. Суидан T. М. Одномерный гравитационно взаимодействующий газ и выпуклая миноранта броуновского движения. - Успехи матем. наук, 2001, т. 56, № 4, с. 7396.

7. E W., Rykov Yu. G., Sinai Ya.G. Generalized variational principles, global weak solutions and behavior with random initial data for systems of conservation laws arising in adhesion particle dynamics. - Comm. Math. Phys., 1996, v. 177, № 2, p. $349-380$.

8. Биллингсли П. Сходимость вероятностных мер. М.: Наука, 1977, 352 с.

Поступила в редакцию 11.VIII. 2003 